\title{
Bioclimatic approach to assessing the potential impact of climate change on wheat midge (Diptera: Cecidomyidae) in North America - CORRIGENDUM
}

\author{
O. Olfert, R.M. Weiss, R.H. Elliott
}

doi:10.4039/tce.2015.40, published by Cambridge University Press, 25 June 2015.

An error was published in Table 1, bottom row of column three. The corrected text "Minimum degree-days above DV0 to complete one generation" (not two generations) is presented in the modified table below.

Table 1. Values for parameter settings for the CLIMEX ${ }^{\circledR}$ model for projecting distribution and relative abundance of Sitodiplosis mosellana in Europe and North America.

\begin{tabular}{|c|c|c|c|}
\hline Index & Parameter & Description & Value \\
\hline \multirow{4}{*}{ Temperature } & DV0 & Limiting low temperature & $5^{\circ} \mathrm{C}$ \\
\hline & DV1 & Lower optimal temperature & $16{ }^{\circ} \mathrm{C}$ \\
\hline & DV2 & Upper optimal temperature & $24{ }^{\circ} \mathrm{C}$ \\
\hline & DV3 & Limiting high temperature & $28^{\circ} \mathrm{C}$ \\
\hline \multirow[t]{4}{*}{ Moisture } & SM0 & Limiting low soil moisture & 0.2 \\
\hline & SM1 & Lower optimal soil moisture & 0.3 \\
\hline & SM2 & Upper optimal soil moisture & 0.9 \\
\hline & SM3 & Limiting high soil moisture & 1.25 \\
\hline \multirow[t]{5}{*}{ Diapause } & DPD0 & Diapause induction day length & 13 \\
\hline & DPT0 & Diapause induction temperature & 3 \\
\hline & DPT1 & Diapause termination temperature & 3 \\
\hline & DPD & Diapause development days & 120 \\
\hline & DPSW & Diapause indicator for winter diapause & 0 \\
\hline \multirow[t]{2}{*}{ Cold stress } & TTCS & Cold stress threshold & $-22{ }^{\circ} \mathrm{C}$ \\
\hline & THCS & Cold stress temperature rate & -0.004 \\
\hline \multirow[t]{2}{*}{ Heat stress } & TTHS & Heat stress temperature threshold & 34 \\
\hline & THHS & Heat stress temperature rate & 0.05 \\
\hline \multirow[t]{2}{*}{ Dry stress } & SMDA & Dry stress threshold & 0.10 \\
\hline & HDS & Dry stress rate & -0.01 \\
\hline \multirow[t]{2}{*}{ Wet stress } & SMWS & Wet stress threshold & 1.5 \\
\hline & HWS & Wet stress rate & 0.0005 \\
\hline \multicolumn{4}{|c|}{ Degree-days above DV0 } \\
\hline & DV0 & & 5 \\
\hline & DV3 & & 30 \\
\hline & MTS & Model step time & 7 \\
\hline \multicolumn{4}{|c|}{ Degree-days above DVCS } \\
\hline & DV3 & & 6.5 \\
\hline & DV4 & & 100 \\
\hline & MTS & Model step time & 7 \\
\hline \multicolumn{4}{|c|}{ Degree-days above DVHS } \\
\hline & DVCS & & 30 \\
\hline & DV4 & & 100 \\
\hline & MTS & & 7 \\
\hline \multicolumn{4}{|c|}{ Degree-days per generation } \\
\hline & PDD & Minimum degree-days above DV0 to complete one generation & 1135 \\
\hline
\end{tabular}

\section{Reference}

Olfert, O., Weiss, R.M., and Elliott, R.H. 2015. Bioclimatic approach to assessing the potential impact of climate change on wheat midge (Diptera: Cecidomyiidae) in North America. The Canadian Entomologist, 148: 52-67. doi:10.4039/tce.2015.40. 